\title{
Cooperative Power Control Scheme for a Spectrum Sharing System
}

\author{
Tae Won Ban, Bang Chul Jung, Member, KIICE
}

\begin{abstract}
In this paper, we investigate a power control problem which is very critical in underlay-based spectrum sharing systems. Although an underlay-based spectrum sharing system is more efficient compared to an overlaybased spectrum sharing system in terms of spectral utilization, some practical problems obstruct its commercialization. One of them is a real-time-based power adaptation of secondary transmitters. In the underlay-based spectrum sharing system, it is essential to adapt secondary user's transmit power to interference channel states to secure primary users' communication. Thus, we propose a practical power control scheme for secondary transmitters. The feedback overhead of our proposed scheme is insignificant because it requires one-bit signaling, while the optimal power control scheme requires the perfect information of channel states. In addition, the proposed scheme is robust to feedback delay. We compare the performance of the optimal and proposed schemes in terms of primary user's outage probability and secondary user's throughput. Our simulation results show that the proposed scheme is almost optimal in terms of both primary user's outage probability and secondary user's throughput when the secondary user's transmit power is low. As the secondary user's transmit power increases, the primary user's outage probability of the proposed scheme is degraded compared with the optimal scheme while the secondary user's throughput still approaches that of the optimal scheme. If the feedback delay is considered, however, the proposed scheme approaches the optimal scheme in terms of both the primary user's outage probability and secondary user's throughput regardless of the secondary user's transmit power.
\end{abstract}

Index Terms - Cognitive Radio, Power Control, Spectrum Sharing, Next-Generation Mobile Communication Systems

\section{INTRODUCTION}

COMMUNICATIONS commission (FCC) introduced the spectrum sharing concept to improve spectral utilization. Based on this concept, secondary users can share spectra dedicated to primary users as long as the interference caused by the secondary users is regulated [2]. For the purpose of the interference regulation, the FCC

Manuscript received August 10, 2011; revised September 4, 2011; accepted September 17, 2011.

Tae Won Ban is with the Mobile laboratory, KT, Seoul, Korea

Bang Chul Jung (Corresponding Author) is with the Dept. of Information and Communication Engineering and the Institute of Marine Industry, Gyeongsang National University, Tongyeong, Korea (Email: bcjung@gnu.ac.kr) also presented several recommendations, one of which is an underlay-based approach. The underlay-based spectrum sharing systems regulate interference caused by secondary users to primary users below a given interference temperature. Compared with an overlaybased concept, the underlay-based approach can dramatically improve spectral efficiency because secondary users can always share primary users' spectrum regardless of whether primary users occupy their spectrum or not. In addition, much higher spectral efficiency can be achieved by opportunistically allocating secondary user's transmit according to primary user's fading channel state information [8]

However, there is a serious drawback in the underlay-based approach in spite of its high spectral efficiency. Secondary users should assess the amount of interference in real-time base and adapt their transmit power based on actual environments. It is believed that this practical obstacle delays the commercialization of the underlay model. Unfortunately, there was, however, no previous work that studied practical power adaptation schemes in spite of this concern of practicality and feasibility on the underlay-based spectrum sharing system. To the best of our knowledge, most of previous studies including our own studies assumed that the real-time based interference measurement and power adaptation is perfect [3-6, 8-11]. Thus, further analysis and study of the power adaptation issue is urgently required to remove the practical obstacle because it can achieve much higher spectral efficiency than the overlaybased approach and is still attractive as a long strategy [12].

On the other hand, recently, the International Telecommunication Unit-Radio Communication Sector (ITU-R) recognized that $\mathrm{CR}$ can be one of promising technologies for the next generation-mobile communication systems and began feasibility studies on $\mathrm{CR}$ over international mobile telecommunication (IMT) systems which are based on infrastructure [1316]. It is expected that next generation mobile communication systems will provide various multimedia contents and services through various radio access technologies. Thus, this infrastructure-based CR can improve the network efficiency, flexibility, and connectivity.

Based on these motivations, in this paper, we investigate a power adaptation problem in the 
underlay-based spectrum sharing system based on infrastructure and propose a practical power control scheme for unlay-based spectrum sharing systems which are based on the cooperation between secondary and primary systems.

The rest of this paper is organized as follows: In Section 2, an infrastructure-based spectrum sharing system model is described and an optimal power control scheme for secondary user is introduced. In Section 3, a cooperative power control scheme is proposed. In Section 4, simulation results are presented to compare the performance of the optimal and proposed schemes. Finally, conclusions are drawn in Section 5

\section{SYSTEM AND CHANNEL MODELS}

\section{A. System Model}

Fig. 1 shows an infrastructure based spectrum sharing system based on an underlay model, where primary and secondary receivers operate on the basis of infrastructure and they can exchange signaling messages to cooperate with each other. Although this signaling message can be exchanged through a wireless or wired link, we assume that they are connected through a wired link because base stations can be clustered to exploit cooperative communication in most of next generation mobile communication systems including 3GPP LTE-advanced [7] and IEEE $802.16 \mathrm{~m}$ [1]. It should be noted that this assumption does not restrict the application of the proposed scheme. Using the notations listed in Table 1 , the received signals at secondary and primary receivers, $\mathrm{ys}_{\mathrm{s}}$ and $\mathrm{yp}_{\mathrm{p}}$ can be described, respectively, as

$$
\begin{gathered}
y_{p}=h_{p p} x_{p}+h_{s p} x_{s}+n \\
y_{s}=h_{s s} x_{s}+h_{p s} x_{p}+n
\end{gathered}
$$

and their received SINRs can be obtained as

$$
\begin{aligned}
& \gamma_{p}=\frac{P_{p}\left|h_{p p}\right|^{2}}{P_{s}^{t x}\left|h_{s s}\right|^{2}+1} \\
& \gamma_{s}=\frac{P_{s}^{t x}\left|h_{s s}\right|^{2}}{P_{p}^{t x}\left|h_{p s}\right|^{2}+1}
\end{aligned}
$$

where we assume that the primary transmitter uses fixed transmit power while the secondary user adapts its transmit power to satisfy a given interference constraint and power is normalized by noise variance without loss of generality.

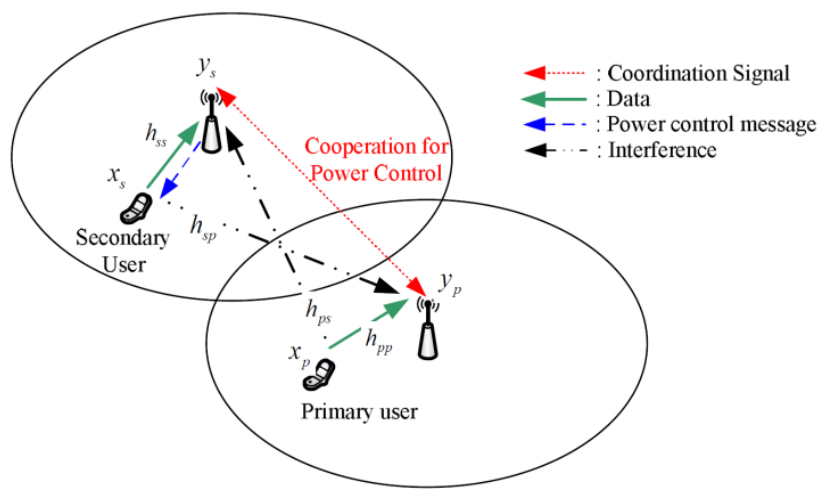

Fig. 1. Infrastructure-based spectrum sharing system

\section{B. Optimal Power Control}

Originally, Ghasemi et al. [1] proposed an optimal power allocation scheme in a fading environment to maximize the ergodic capacity of the secondary user given an interference temperature constraint at the primary receiver, which was also described in Eq. (1). They assumed that a secondary transmitter can obtain perfect information of the channel from secondary transmitter to primary receiver, $h_{s p}$. However, the power allocation scheme cannot exploit the fading effect of the channel from primary transmitter to primary receiver, $h_{p p}$. Fig. 2 shows the channel gains and the secondary user's transmit power. High channel gain value of the interference channel seriously decreases secondary transmitter's transmit power although the primary user's channel is good enough to tolerate higher interference from the secondary user. It is desirable to allow secondary user to use higher transmit power when the primary user's channel gain is good enough to satisfy its target SINR value in spite of the interference from the secondary user. Thus, the secondary user's performance can be improved if the secondary user can obtain the channel state information of the primary user, $h_{p p}$ and exploit the fading effect of the channel [8]. Based on this fact, the optimal transmit power of the secondary transmitter can

\begin{tabular}{|c|c|c|}
\hline Parameters & Description & Value \\
\hline$x_{p}$ & Transmitted signal from a primary transmitter & \\
\hline$x_{s}$ & Transmitted signal from a secondary transmitter & \\
\hline$P_{p}$ & Primary user's available transmit power $[\mathrm{dB}]$ & \\
\hline$P_{s}$ & Secondary user's available transmit power $[\mathrm{dB}]$ & \\
\hline$P_{s}^{t x}$ & Secondary user's transmit power $[\mathrm{dB}]$ & \\
\hline$y_{p}$ & Received signal at a primary receiver & \\
\hline$y_{s}$ & Received signal at a secondary receiver & \\
\hline$h_{i j}$ & $\begin{array}{l}\text { Channel gain from transmitter } i \text { to receiver } j \\
(i \text { and } j \text { can be primary, } p \text { or secondary, } s)\end{array}$ & $\mathcal{C} \mathcal{N}\left(0, \sigma_{i j}^{2}\right)$ \\
\hline$\gamma_{p}$ & Primary user's received SINR & \\
\hline$\gamma_{s}$ & Secondary user's received SINR & \\
\hline$n$ & AWGN at a receiver & $\mathcal{C N}\left(0, \sigma^{2}\right)$ \\
\hline$\Gamma$ & Primary user target SINR $[\mathrm{dB}]$ & \\
\hline
\end{tabular}
be obtained as a function of $h_{p p}$, as shown in Fig. 3.

TABLE I

NOTATIONS 


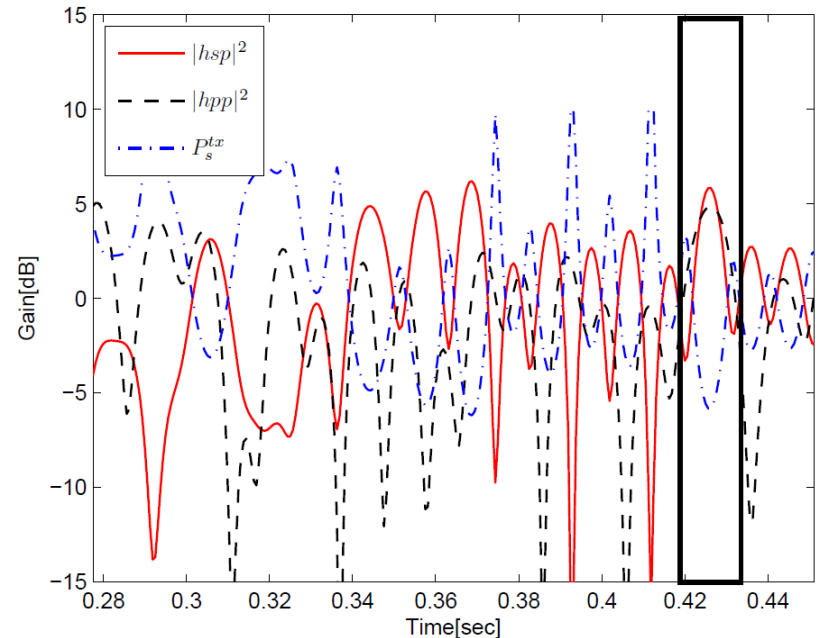

Fig. 2. Channel gains and secondary user's transmit power

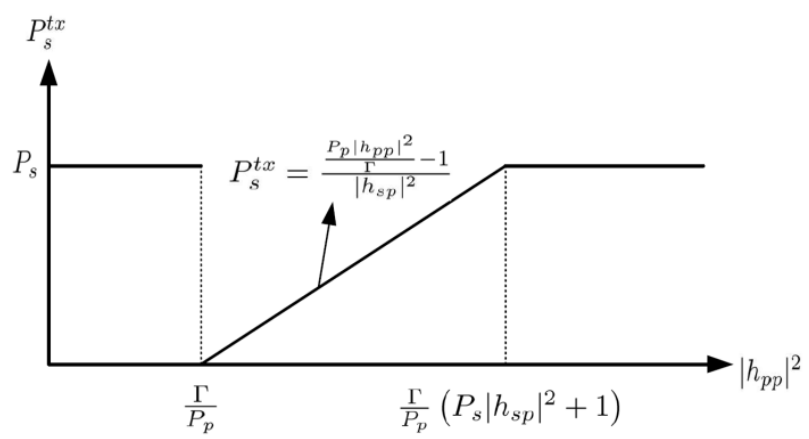

Fig. 3. Optimal power control scheme of secondary user

- When $\left|h_{p p}\right|^{2}<\frac{\Gamma}{P_{p}}$ the primary user's target SINR can not be satisfied because of its own deep fading even if the secondary user does not transmit. In this case, it is optimal for secondary user to transmit its data using its peak transmit power, Ps.

- When $\frac{\Gamma}{P_{p}} \leq\left|h_{p p}\right|^{2}<\frac{\Gamma}{P_{p}}\left(P_{s}\left|h_{s p}\right|^{2}+1\right)$, the secondary transmitter should adaptively reduce its transmit power so that the primary user's target SINR is satisfied as follows:

$$
\gamma_{s}=\frac{P_{p}\left|h_{p p}\right|^{2}}{P_{s}^{t x}\left|h_{s p}\right|^{2}+1}=\Gamma
$$

Then, the secondary user's optimal transmit power is determined as

$$
P_{s}^{t x}=\frac{\frac{P_{p}\left|h_{p p}\right|^{2}}{\Gamma}-1}{\left|h_{s p}\right|^{2}}
$$

which is linearly proportional to the value of $\left|h_{p p}\right|^{2}$. The secondary user should have the prior information of channel states, $\left|h_{p p}\right|^{2}$ and $\left|h_{p p}\right|^{2}$ to compute $\left|h_{p p}\right|^{2}$. It should be noted that $P_{s}^{t x}$ can be obtained by measuring the pilot channel from the primary receiver, while $\left|h_{p p}\right|^{2} \quad$ can only obtained through a direct feedback from the primary receiver.

- When $\frac{\Gamma}{P_{p}}\left(P_{s}\left|h_{s p}\right|^{2}+1\right) \leq\left|h_{p p}\right|^{2}$, the primary user's target SINR can be unconditionally satisfied regardless of the secondary user's transmission because the channel state is good enough to tolerate the secondary user's peak power transmission. Thus, the secondary user can transmit its data using its peak power, Ps.

\section{ANALYSIS OF OUTAGE PROBABILITY}

Although the optimal transmit power of secondary transmitters can be determined as described in 2.2, it may be impractical because it requires direct communication between the primary receiver and the secondary transmitter for the feedback of channel state information, which is prohibitive in spectrum sharing systems because it can cause a serious burden to primary users. Thus, we propose an empirical power control scheme for secondary users, which is based on the cooperation between secondary and primary infrastructures. Fig. 4 shows the flow diagram of our proposed scheme, where $\Delta_{u}$ and $\Delta_{d}$ denote the power increment and decrement steps of secondary transmitters, respectively.

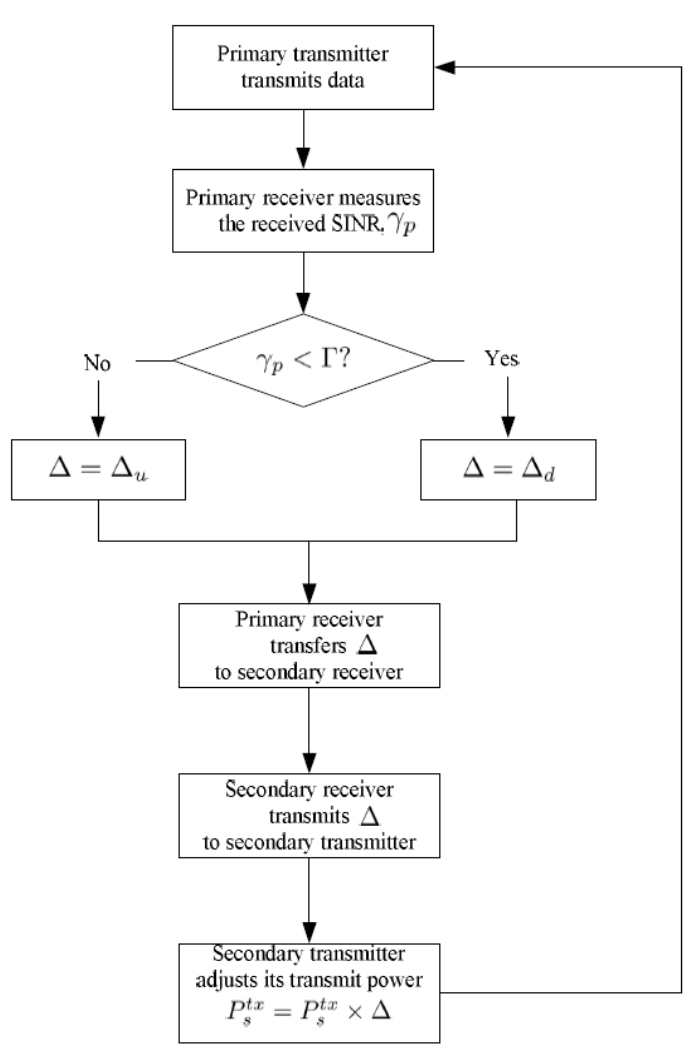

Fig. 4. Proposed power control scheme for a secondary user 
Although the primary receiver is assumed to transfer the values of $\Delta_{d}$ and $\Delta_{u}$ to the secondary transmitter through the secondary receiver, they can be replaced with one bit indicator to reduce feedback overhead if the values of $\Delta_{u}$ and $\Delta_{d}$ are shared between the secondary transmitter and the primary receiver.

\section{SIMULATION RESULTS}

Unfortunately, it is intractable to mathematically analyze the performance of the proposed scheme. Thus, we present simulation results to investigate the proposed power control scheme and compare the performance of the proposed scheme with that of the optimal power control scheme in terms of primary user's outage probability and secondary user's throughput, which are defined, respectively, as

$$
\begin{aligned}
P_{p}^{\text {out }} & =\operatorname{Pr}\left[\gamma_{p}<\Gamma\right] \\
T h_{s} & =\mathbb{E}\left[\log _{2}\left(1+\gamma_{s}\right)\right]
\end{aligned}
$$

We assume that all average channel gains are equal to 1 to focus on the effect of small-scale fading, although the effect of large-scale channel fading can be incorporated by considering adequate average channel gains. The simulation parameters are summarized in Table II.

TABLE II

\section{SIMULATION PARAMETERS}

\begin{tabular}{|c|c|c|}
\hline Parameters & Description & Value \\
\hline \hline$\Gamma$ & Primary user target SINR $[\mathrm{dB}]$ & 0 \\
$v$ & User velocity & $30 \mathrm{~km} / \mathrm{h}$ \\
$f$ & Carrier frequency & $2 \mathrm{GHz}$ \\
$c$ & Light velocity & $3 \times 10^{8} \mathrm{~m} / \mathrm{s}$ \\
$f_{d}$ & Doppler shift & $\frac{v f}{c}$ \\
$\Delta_{u}$ & Power increment step $[\mathrm{dB}]$ & 1 \\
$\Delta_{d}$ & Power decrement step $[\mathrm{dB}]$ & -3 or -1 \\
$f_{p c}$ & Power control frequency $[\mathrm{Hz}]$ & 800 or 1600 \\
$d_{f b}$ & Feedback delay [sec] & 1 sample $=\frac{1}{f_{p c}}$ \\
\hline
\end{tabular}

Fig. 5 shows the primary user's outage probability and secondary user's throughput when $P_{s}=20 \mathrm{~dB}, v=30 \mathrm{~km} / \mathrm{h}, \quad \Delta_{u}=1 \mathrm{~dB}, \Delta_{d}=-1 \mathrm{~dB}$ and, $f_{p c}=800 \mathrm{~Hz}$, The optimal power control scheme serves as theoretical upper-bound for the proposed scheme in terms of both primary user's outage and secondary user's throughput. As derived in Appendix D, the primary user's outage probability of the optimal scheme is independent of the secondary user's transmit power because the secondary user can always compute its optimal transmit power to secure the primary user's transmission if it can obtain the perfect channel state information. When the secondary user's transmit power is low, the proposed scheme approaches the optimal scheme in terms of both the primary user's outage probability and secondary user's throughput. As the secondary user's transmit power increases, the primary user's outage probability of the proposed scheme is degraded, while the secondary user's throughput of the proposed scheme is still optimal. In practice, the feedback of channel state information always accompanies with feedback delay. If the feedback delay is considered, the primary user's outage probability of the optimal power control scheme is degraded due to the inaccurate channel state information, while the secondary user's throughput is not degraded. On the other hand, the proposed scheme is very robust to the feedback delay and almost optimal regardless of the secondary user's transmit power in terms of both primary user's outage probability and secondary user's throughput.

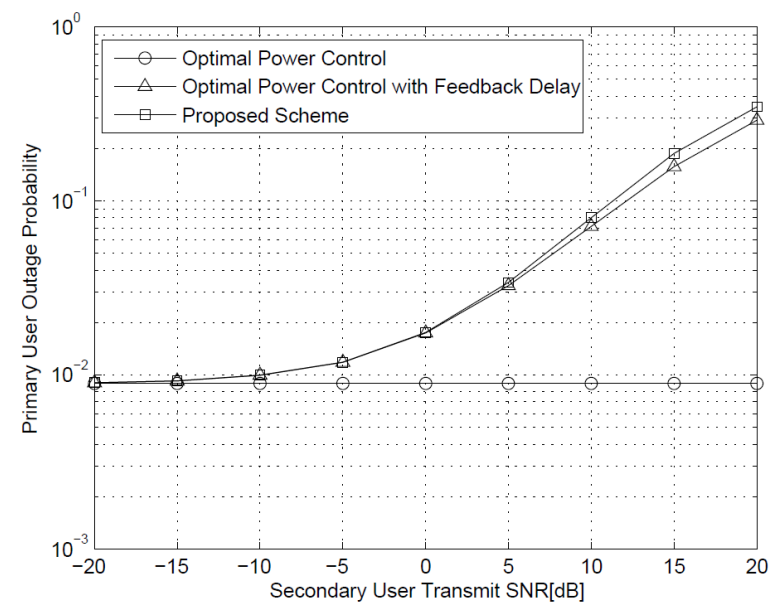

(a)

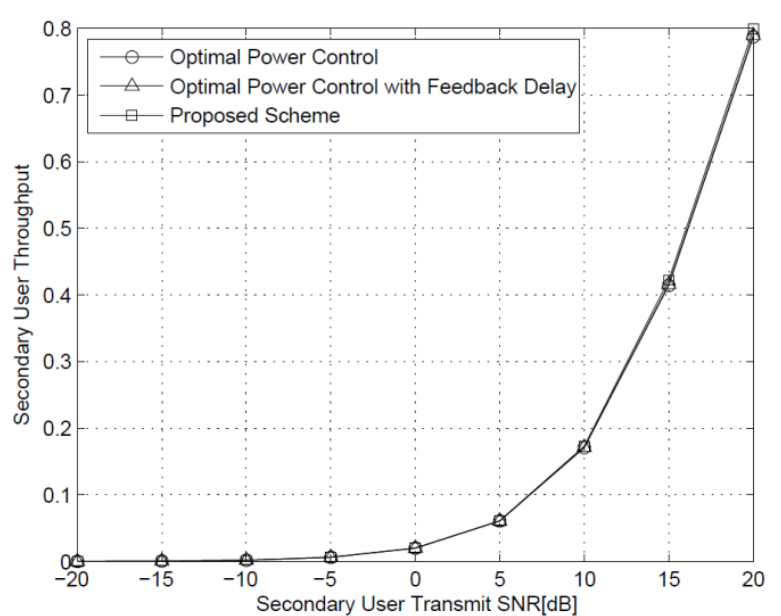

(b)

Fig. 5. Primary user's outage probability and secondary user's throughput when $P_{s}=20 \mathrm{~dB}, v=30 \mathrm{~km} / \mathrm{h}, \quad \Delta_{u}=1 \mathrm{~dB}$. $\Delta_{d}=-1 \mathrm{~dB}$ and $f_{p c}=800 \mathrm{~Hz}$

(a) Primary user's outage Probability

(b) Secondary user's throughput 
Fig. 6 shows the performance of both schemes when $f_{p c}=1600 \mathrm{~Hz}$ and all other parameters are the same as in Fig. 5. As the frequency of power control is doubled, the performance is slightly improved in terms of primary user's outage probability. However, the performance improvement is insignificant if the increased power control overhead is considered.

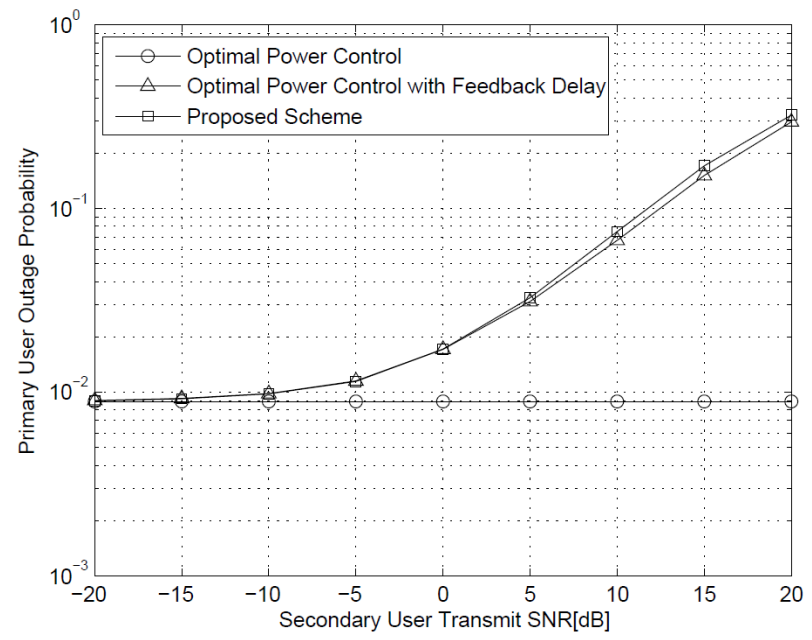

(a)

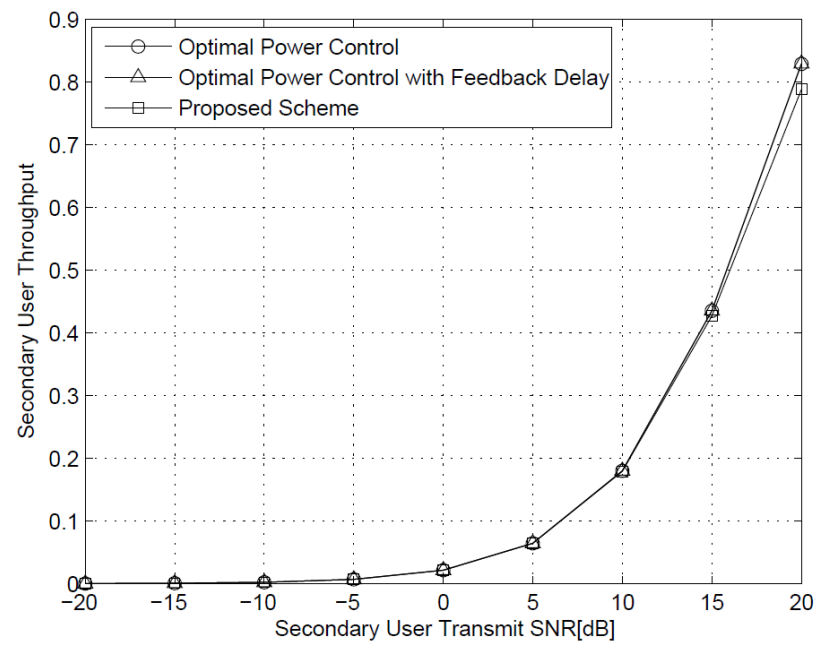

(b)

Fig. 6. Primary user's outage probability and secondary user's throughput when $P_{s}=20 \mathrm{~dB}, v=30 \mathrm{~km} / \mathrm{h}, \quad \Delta_{u}=1 \mathrm{~dB}$, $\Delta_{d}=-1 \mathrm{~dB}$ and $f_{p c}=1600 \mathrm{~Hz}$

(a) Primary user's outage Probability

(b) Secondary user's throughput

Fig. 7 shows the performance of both schemes when $\Delta_{d}=-3 \mathrm{~dB}$ and all other parameters are the same as in Fig. 5. It is shown that the primary user's outage probability can be greatly improved at the cost of secondary user's throughput. That is, secondary user should aggressively reduce its transmit power to secure primary user's communication when primary user is in outage state although its own throughput can be degraded. It should be noted that in order to improve the primary user's outage probability, the adjustment of $\Delta_{d}$ is much more efficient than the increase in the power control frequency. In addition, the adjustment of $\Delta_{d}$ does not cause any additional overhead while the increase in the power control frequency linearly increases signaling overhead.

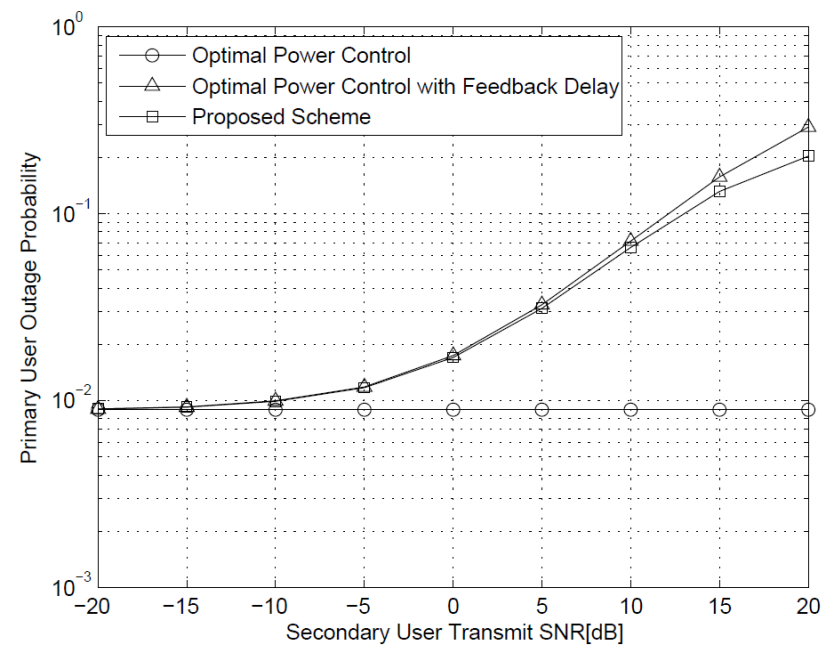

(a)

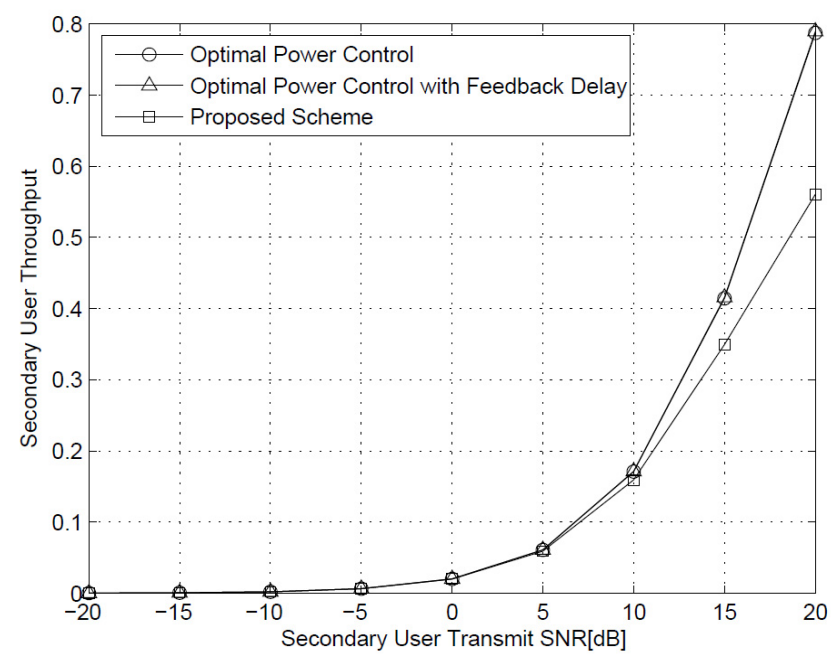

(b)

Fig. 7. Primary user's outage probability and secondary user's throughput when $P_{s}=20 \mathrm{~dB}, v=30 \mathrm{~km} / \mathrm{h}, \quad \Delta_{u}=1 \mathrm{~dB}$, $\Delta_{d}=-3 \mathrm{~dB}$ and $f_{p c}=800 \mathrm{~Hz}$

(a) Primary user's outage Probability

(b) Secondary user's throughput 


\section{SUMMARY}

In this paper, we proposed a practical power control scheme for secondary users in an infrastructure-based underlay spectrum sharing system. Although the underlay-based spectrum sharing system can dramatically improve the spectral efficiency compared to the overlay model, it is challenging to regulate the interference caused by secondary users. Specially, it is very difficult to estimate interference channels and adapt secondary users' transmit power in real-time base. In spite of these practical obstacles, most of previous studies assumed that the channel estimation and real-time power adaptation are perfect. Thus, we proposed an empirical power control scheme based on an infrastructure where a primary receiver estimates its received SINR and transfers power control message to a secondary transmitter through a secondary receiver. Our simulation results show that the primary user's outage probability of the proposed scheme approaches that of the optimal scheme when the secondary user's transmit power is low and the proposed scheme is almost optimal in terms of the secondary user's throughput regardless of the secondary user's transmit power. It is also shown that our proposed scheme is very robust to the feedback delay, while the optimal power control scheme is very vulnerable to the feedback delay. If the feedback delay is considered, our proposed scheme yields almost optimal performance in terms of both primary user's outage probability and secondary user's throughput. It should be also noted that our proposed scheme requires one-bit signaling to control secondary user's transmit power while the optimal power control scheme requires full channel state information of both secondary and primary users.

\section{ACKNOWLEDGEMENT}

This research was supported by Basic Science Research Program through the National Research Foundation of Korea (NRF) funded by Ministry of Education, Science, and Technology (2010-0011140).

\section{REFERENCES}

[1] IEEE 802.16m System Requirements, IEEE Std. 802.16m-07/002r5, August 2008.

[2] Federal Communications Commision, "Spectrum policy task force report, ET Docket No.02-135," November 2002.

[3] M. Gastpar, "On capacity under received-signal constraints," Proc. of 42nd Annual Allerton Conf. Comm., Control Comp., September 2004.

[4] - "On capacity under receiver and spatial spectrum-sharing constraints," IEEE Trans-actions on Information Theory, vol. 53, no. 2, pp. 471-487, February 2007. .

[5] A. Ghasemi and E. S. Sousa, "Fundamental limits of spectrumsharing in fading environments," IEEE Transactions on Wireless Communications, vol. 6, no. 2, pp. 649-658, February 2007.
[6] R. Zhang and Y.-C. Liang, "Exploiting multi-antennas for opportunistic spectrum sharing in cognitive radio networks," IEEE Journal of Selected Topics in Signal Processing, vol. 2, no. 1, pp. 88-102, February 2008.

[7] Evolved Universal Terrestrial Radio Access (E-UTRA) and Evolved Universal Terrestrial Radio Access Network (EUTRAN);Overall description; Stage 2, 3GPP Std. TS 36.300, September 2008.

[8] Y. Chen, G. Yu, Z. Zhang, H.-H. Chen, and P. Qiu, "On cognitive radio networks with opportunistic power control strategies in fading channels," IEEE Transactions on Wireless Communications, vol. 7, no. 7, pp. 2752-2761, July 2008.

[9] T. W. Ban, D. K. Sung, B. C. Jung, and W. Choi, "Capacity analysis of an opportunistic scheduling system in a spectrum sharing environment," Proc. of IEEE Globecom, November 2008.

[10] T. W. Ban, W. Choi, B. C. Jung, and D. K. Sung, "Multiuser diversity in a spectrum sharing system," IEEE Transactions on Wireless Communications, vol. 8, no. 1, pp. 102-106, January 2009.

[11] T.W. Ban, W. Choi, and D. K. Sung, "Capacity and energy efficiency of multi-user spectrum sharing systems with opportunistic scheduling," IEEE Transactions on Wireless Communications, vol. 8, no. 6, pp. 2836-2841, June 2009.

[12] Federal Communications Commision, "Spectrum policy task force report, ET Docket No.03-237."

[13] Hae Young Lee, "Standardization of CR in ITU-R," TTA Journal, no. 124, pp. 86-91, August 2009.

[14] ITU-R Working Party 1B, "Report of the 2nd meeting of Working Party 1B (Seoul, 25 February - 4 March 2009)."

[15] ITU-R Working Party 5D, "Report of the 4th meeting of Working Party 5D (Geneva, 10-17 February 2009)."

[16] ITU-R Working party 5A, "Working document towards a preliminary draft new report $<$ cognitive radio systems in the land mobile service>."

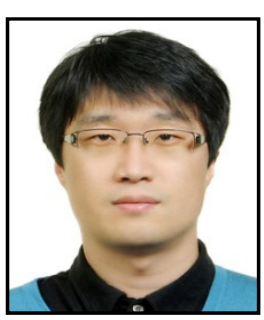

Tae Won Ban received the B.S. and M.S. degrees in electronics engineering from Kyungpook National University, Korea, in 1998 and 2000, respectively, and the Ph.D. degree in electrical engineering from the Korea Advanced Institute of Science and Technology (KAIST), Korea, in 2010. Since January 2000 , he has been working for Korea Telecom, Korea. His research interests include OFDM, MIMO, radio resource management for mobile communication systems, cognitive radio, and relay systems.

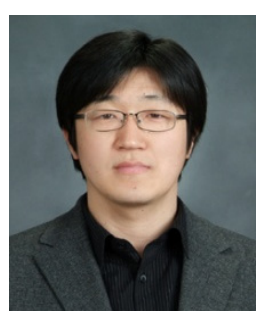

Bang Chul Jung received the B.S. degree in Electronics Engineering from Ajou University, Suwon, Korea, in 2002 and the M.S. and Ph.D degrees in Electrical \& Computer Engineering from Korea Advanced Institute of Science and Technology (KAIST), Daejeon, Korea, in 2004 and 2008, respectively. He was a research professor with KAIST Institute for Information Technology Convergence, Daejeon, Korea, until Feb. 2010. He is now an assistant professor of department of Information and Communication Engineering, Gyeonsang National University, Korea.

Dr. Jung is a member of IEEE, IEICE, Marquis Who's Who in the World (2011 Edition), IBC Top 100 Engineers (2011 Edition). He was the recipient of the Bronze Prize in Intel Student Paper Contest in 2005, the First Prize in Research Performance Evaluation System (RPES) for Doctorial Student (School of EECS, KAIST) in 2008, the First Prize in KAIST's Invention Idea Contest in 2008, the Bronze Prize in Samsung Humantech Paper Contest in 2009, the IEEE Communication Society Asia-Pacific Outstanding Young Researcher Award in 2011. 